\title{
IDENTIFICACIÓN Y \\ CARACTERIZACIÓN DE LOS \\ TRABAJADORES POBRES EN \\ COLOMBIA, 2002-2012
}

Roberto Mauricio Sánchez Torres*

$\mathrm{E}^{1}$ 1 término "pobreza” se refiere a un problema que abarca diferentes aspectos. Las condiciones de vida, el acceso a servicios sociales básicos, las características de la vivienda, la dependencia económica y el nivel de ingreso son aspectos y maneras de medir la pobreza (GEEP, 2007). La pobreza afecta a buena parte de la población latinoamericana; a más de la mitad en países de Centroamérica como Guatemala $(54,8 \%)$, Honduras $(67,4 \%)$ y Nicaragua $(58,3 \%)$, y a una proporción menor en países del Cono Sur como Argentina (8,6\%), Uruguay (8,6\%) y Chile (11,5\%) (Cepal, 2013).

Como la pobreza es una característica de los hogares, son pobres los miembros de hogares pobres ${ }^{1}$, independientemente de que sean inactivos, desempleados o empleados, aunque la incidencia es mayor en hogares con alta dependencia económica (Ponthieux y Concialdi, 2001). En América Latina, donde buena parte del empleo es informal con relaciones laborales endebles, y hay alta proporción de empleos por cuenta propia, prolifera el empleo al margen de la regulación y con baja remuneración, la pobreza tiene gran incidencia entre los trabajadores, y no solo afecta a quienes no participan en el mercado laboral y a los desempleados (Majid, 2001).

Según cálculos del Dane, en 2012 la pobreza era en Colombia del 24,8\% por necesidades básicas insatisfechas, del 27,0\% según el

* Economista, Universidad Nacional de Colombia. Becario del Centro de Estudios e Investigaciones Laborales, Conicet-Argentina, [rmsanchezt@gmail.com]. Fecha de recepción: 10 de febrero de 2014, fecha de modificación: 28 de julio de 2014, fecha de aceptación: 29 de abril de 2015. Sugerencia de citación: Sánchez T., R. M. "Identificación y caracterización de los trabajadores pobres en Colombia, 2002-2012", Revista de Economía Institucional 17, 32, 2015, pp. 295-315. DOI: 10.18601/01245996.v17n32.10

${ }^{1}$ Aunque a veces la referencia sea la vivienda y no los hogares, como cuando se mide por necesidades básicas insatisfechas. 
za (Dane, 2013a). Sea cual sea la manera de medirla, la pobreza se redujo notablemente en el país durante la última década, sobre todo en los últimos años; del 49,7\% en 2002 bajó al 40,3\% en 2009 y al 32,9\% en 2012 (MESEP, 2012). Pero el mercado laboral no muestra resultados similares: la tasa anual de desempleo solo descendió a un dígito en 2013 y la informalidad afecta a más de la mitad de los trabajadores; entre 2002 y 2010 el 40\% de la variación neta (aumento) del empleo urbano fue informal (en el sector informal o sin relación laboral formal) y el $65 \%$ de la variación del empleo nacional total fue en empleos independientes (Sánchez, 2013).

La aparente divergencia entre la mejora del nivel de vida de la población de mayores carencias y la persistencia de problemas en el mercado laboral, como la informalidad y el desempleo, justifica el objetivo de este artículo: analizar la pobreza vinculada a ciertas formas de inserción laboral y a ciertas actividades y sectores de actividad. Para ello se identifica y caracteriza a los trabajadores de hogares pobres, para determinar de qué modo la reducción de la pobreza ha alterado la importancia de ciertas características personales y ocupacionales en la inserción laboral de los miembros de hogares que se mantienen en una situación de pobreza.

En la primera sección se hace una breve referencia a los enfoques para detectar y medir la pobreza y se precisa qué son trabajadores de hogares pobres. En la segunda sección se indican las fuentes de información, el enfoque metodológico y la definición de los principales indicadores. La tercera sección compara los principales indicadores de pobreza de la población total y de los trabajadores, y en la siguiente se presenta su descomposición regional. En la quinta sección se caracteriza a los trabajadores pobres y se estima la probabilidad de ser trabajador informal teniendo en cuenta variables personales, ocupacionales, de rama de actividad y área geográfica. En la parte final se presentan las conclusiones.

\section{POBREZA Y TRABAJADORES DE HOGARES POBRES}

Los enfoques para estudiar y medir la pobreza son diversos y dependen del contexto económico, histórico o social. Para identificar la población pobre se adoptan algunos criterios y se especifican las variables correspondientes para determinar si un hogar es o no pobre. En la medición unidimensional se toma el ingreso o el consumo como variable de referencia y en mediciones multidimensionales se toman diversos aspectos, como las características de las viviendas, el nivel de dependencia económica, la asistencia escolar de los menores, la salud, 
las condiciones de trabajo, el tipo de inserción laboral y el bienestar subjetivo (GEEP, 2007; Alkire y Foster, 2009).

Una característica esencial de las principales nociones de pobreza es que la unidad de análisis son los hogares y no los individuos, de modo que un individuo es pobre si forma parte de un hogar pobre. Así, quien cumple o no el umbral de referencia es el hogar (unidad de ingreso o gasto). Los trabajadores pobres son aquellos que trabajan y son miembros de hogares pobres; por ello, el concepto de trabajador pobre interrelaciona dos unidades de análisis: el individuo, que cumple el criterio de estar o no empleado, y el hogar, la unidad a la que se atribuye la condición de pobre. En la identificación de los trabajadores pobres se conjugan dos situaciones (no en forma unívoca ni directa): en el individuo empleado, la situación de bajos salarios (ingresos) ${ }^{2}$; $y$ en el hogar, la alta dependencia económica, es decir, la baja proporción empleados/número de miembros.

La relación entre el mercado laboral y el nivel de pobreza se ha analizado desde diferentes perspectivas. De acuerdo con Majid (2001), esta relación depende del nivel de desarrollo y de las características del sistema de seguridad social. En los países en desarrollo donde no hay un sistema de seguridad social establecido, el salario mínimo y la organización de los trabajadores no funcionan o no incluyen amplios sectores de trabajadores, hay formas híbridas de inserción laboral (no asalariadas, sin remuneración, relaciones laborales endebles, vínculos serviles, etc.), se presentan dos situaciones en el mundo del trabajo: una gran proporción de los pobres debe trabajar en ocupaciones de muy baja remuneración, y los desempleados no son siempre pobres, en la medida en que consiguen, mediante canales institucionales pero sobre todo por vínculos personales (familia, redes sociales), niveles de bienestar (ingreso o consumo) superiores a los umbrales de pobreza.

Otros enfoques resaltan el papel del crecimiento económico. Fields muestra que en la mayoría de los países en desarrollo el crecimiento genera empleo, aumenta los ingresos laborales y por esa vía reduce la pobreza, pero no garantiza mejoras en la distribución del ingreso ni la calidad y permanencia del empleo. Aunque el crecimiento económico es un elemento fundamental para reducir la pobreza, no es suficiente, y se necesitan políticas sociales, laborales y de redistribución del

${ }^{2}$ En algunos enfoques la situación laboral y la pobreza se vinculan a un bajo
nivel de remuneraciones y no directamente al estatus de pobreza del hogar corres-
pondiente. Para Marx y Verbist (1998) un trabajador con bajo salario es aquel que
trabaja tiempo completo todo el año y recibe menos del $66 \%$ del salario mediano
de todos los trabajadores de tiempo completo en ese año. Y proponen una medida
relativa de la pobreza: es pobre un hogar cuyo ingreso familiar equivalente es un
$50 \%$ menor que el promedio del ingreso familiar equivalente del total de hogares. 
ingreso (Fields, 2012; Cruces y Gasparini, 2013). Para otros autores un objetivo de la política pública debe ser mejorar la situación laboral; Cecchini y Uthoff (2008) argumentan que se necesita mejorar la calidad del empleo y aumentar la productividad del trabajo para que el crecimiento económico se refleje en una reducción de la pobreza y en mejores indicadores sociales.

En los países desarrollados de altos ingresos donde la pobreza se suele medir en términos relativos, la relación entre pobreza y desempleo es más clara, buena parte de los individuos de la cola inferior de la distribución están desempleados, pero la existencia de un piso de seguridad social y el mayor ingreso familiar hacen posible que no acepten empleos muy mal remunerados (Marx y Verbist, 1998).

En América Latina, la relación entre pobreza y mercado laboral depende más de la situación del empleo y del tipo de inserción laboral (informalidad, precariedad, trabajo por cuenta propia, bajos salarios, inseguridad, inestabilidad, etc.) que del alto nivel de desempleo, aunque esto es más cierto para algunos países y determinados grupos de población ${ }^{3}$. En países de ingreso bajo y medio, las personas que no consiguen empleo y carecen de protección social no pueden decidir si trabajan o no, y para obtener ingresos y suplir sus necesidades mínimas suelen emplearse en ocupaciones por cuenta propia de baja productividad o de bajos salarios e inadecuadas condiciones de trabajo (Banerjee y Duflo, 2011).

La reducción de la pobreza que se presentó en América Latina desde comienzos de los años noventa hasta mediados de la década anterior obedece en su mayor parte al aumento de la participación laboral de los miembros de los hogares y a la reducción de la tasa de dependencia (Cecchini y Uthoff, 2008); entre 1997 y 2007 el 79\% de la reducción de la pobreza en la región se debió al crecimiento medio del ingreso y el 21\% restante a su redistribución (Medina y Galván, 2014). Los estudios sobre pobreza se centran en la población total teniendo en cuenta los hogares, y pocos examinan la relación entre pobreza e inserción laboral, tipo de empleo y rama de actividad. Para llenar ese vacío aquí se estudia la pobreza de los trabajadores tomando como referencia su situación laboral y las características de su ocupación, con el fin de establecer la relación entre la reducción de la pobreza y los cambios en el tipo de empleo, las características ocupacionales, la inserción laboral y el tipo de unidad económica.

${ }^{3}$ Según Fields (2012), en el mundo el problema del empleo es mayor que el del desempleo: mientras que hay 200 millones de desempleados, 900 millones de trabajadores tienen un ingreso tan bajo que no cubre las condiciones de vida mínimas. 


\section{DATOS, METODOLOGÍA E INDICADORES DE POBREZA}

Los datos que se utilizan provienen de la Encuesta Continua de Hogares, 2002 y 2005, y la Gran Encuesta Integrada de Hogares, 2008 y 2012, realizadas por el Dane. En 2006 hubo un cambio metodológico en la encuesta de hogares, y la Misión para el Empalme de las Series de Empleo, Pobreza y Desigualdad (MESEP) tomó los datos de pobreza monetaria y empalmó los umbrales de pobreza de cada hogar y el ingreso familiar total. Se dispone de las series empalmadas entre 2002 y 2010 (no hay microdatos para 2006 y 2007) y los datos de 2011 y 2012 que siguen la metodología propuesta por la MESEP, de modo que las encuestas que se toman como fuente de información tienen un alto grado de comparación, aunque no se realicen con la misma metodología.

Debido a la disponibilidad de datos, a los objetivos y a que es la manera más usual en América Latina, aquí medimos la pobreza considerando el ingreso; en particular, contrastamos el ingreso familiar per cápita con los umbrales de pobreza per cápita para identificar si una persona es pobre o no. Se toma como referencia el umbral de pobreza estimado por el Dane, que tiene en cuenta las necesidades alimentarias basadas en la Encuesta Nacional de Ingresos y Gastos (2006-2007), y las necesidades no alimentarias determinadas por el coeficiente de Orshansky, que indica la proporción entre gasto total y gasto en alimentos. Las líneas de pobreza calculadas por el Dane tienen en cuenta las diferencias regionales de precios, acceso a alimentos y niveles de consumo de los grupos de referencia para estimar el coeficiente de Orshansky. Por lo tanto, dependen del departamento y de si es una zona urbana o rural.

En las dos secciones siguientes se examina la pobreza tomando en cuenta cuatro indicadores muy utilizados en los estudios sobre pobreza, los indicadores FGT (Foster, Greer y Thorbecke, 1984) y el indicador de Sen. Esta familia de indicadores forma parte de los "índices generalizados de brecha de pobreza", y se construyen comparando la línea de referencia y la variable observada (ingreso) (Gasparini et al., 2013).

$F G T(\propto)=\frac{1}{N} \sum_{i=1}^{N} k_{i}\left(1-\frac{x_{i}}{z_{i}}\right)^{\alpha} ; \begin{aligned} & k_{i}=1 \text { si } x_{i}<z_{i}, \propto \geq 0 \\ & k_{i}=0 \text { si } x_{i} \geq z_{i}\end{aligned}$

donde $N$ es el número de individuos (población total o total de empleados, según sea el caso), $x_{i}$ el ingreso del individuo $i$ (aquí el ingreso per cápita familiar), $z_{i}$ el umbral de pobreza de referencia para el individuo $i, k_{i}$ el indicador de los individuos pobres (cuyo ingreso 
es menor que el umbral de pobreza) y $\propto$ el parámetro que indica el tipo de indicador FGT y la ponderación de cada individuo pobre. E1 umbral de pobreza $\left(z_{i}\right)$ no indica que se haya construido una línea de pobreza equivalente para cada individuo, solo resalta que no hay una línea de pobreza única; como ya se señaló, las líneas de pobreza del Dane dependen del departamento y de la zona geográfica. En 2012 había 300 líneas de pobreza diferentes: en promedio, la línea de pobreza per cápita en las 13 ciudades principales era de 222.949 pesos de ese año, en el resto urbano de 223.406 pesos y en las zonas rurales de 133.534 pesos.

Cuando $\alpha=0$, el FGT es la proporción de pobres o incidencia de la pobreza, es decir, la proporción de la población que está por debajo del umbral de pobreza $\left(z_{i}\right)$. El análisis de la pobreza en términos de incidencia, número y proporción de pobres es sencillo, pero no tiene en cuenta lo que sucede en el margen, la distancia entre los individuos pobres y el umbral de pobreza ni la distribución dentro de la población pobre. Cuando $\alpha=1$, el FGT se conoce como brecha de pobreza, y tiene en cuenta la distancia entre el ingreso de los individuos pobres y su línea de pobreza. Este indicador computa la tasa de incidencia según la brecha de pobreza del promedio de personas pobres; por lo tanto, no solo es función del número de pobres sino también de la distancia entre ese ingreso promedio y el umbral de pobreza (Foster et al., 1984).

A medida que $\alpha$ aumenta es mayor la importancia de los individuos más alejados del umbral de pobreza de referencia en el cálculo del FTG. En general, se toma un $\alpha=2$ como indicador de intensidad de la pobreza; este indicador cambia aunque la proporción de pobres o la brecha de pobreza promedio no varíen, lo que puede ocurrir si hay transferencias entre personas pobres. Además, se considera un indicador que tiene en cuenta la proporción de pobres, la brecha promedio entre su ingreso y el umbral de pobreza, y la desigualdad entre los pobres medida por el coeficiente de Gini $\left(G^{p}\right)$, conocido como el Indicador de Sen, y su particularidad es que tiene en cuenta la distribución de ingresos dentro de la población pobre (Gasparini, et al., 2013).

Indicador de Sen $=\frac{1}{N} \sum_{i=1}^{N} k_{i} G^{p}+\frac{1}{N} \sum_{i=1}^{N} k_{i}\left(1-\frac{x_{i}}{z_{i}}\right)\left(1-G^{p}\right) ; \begin{aligned} & k_{i}=1 \text { si } x_{i}<z_{i} \\ & k_{i}=0 \text { si } x_{i} \geq z_{i}\end{aligned}$ 


\section{INDICADORES DE POBREZA, POBLACIÓN TOTAL Y EMPLEADOS}

La pobreza se redujo en forma significativa durante la última década, medida con cualquier indicador convencional. El cuadro 1 muestra los indicadores FGT de incidencia $(\alpha=0)$, brecha $(\alpha=1)$ e intensidad $(\alpha=2)$ y el Indicador de Sen para la población total y la empleada en el período 2002-2012. La incidencia se redujo en unos 16 puntos, 10 de ellos entre 2008 y 2012. La brecha también disminuyó en la primera década de este siglo, y en forma más notable en los últimos cinco años. La intensidad, que tiene en cuenta el número de pobres y la distancia al umbral de pobreza, y da mayor ponderación a los más pobres, también tendió a disminuir en forma sistemática ${ }^{4}$. La reducción del 38\% en el Indicador de Sen es el resultado de la reducción conjunta de la incidencia y la brecha de pobreza cuando se tienen en cuenta los cambios en la distribución del ingreso entre los pobres.

La pobreza también tiende a reducirse para la población que tiene empleo, que no incluye personas inactivas, desempleadas y menores de 12 años. Es evidente que si solo se toma en cuenta a los trabajadores se excluye a la población que no tiene ingresos laborales, y a todos los hogares que no tienen miembros que participen en el mercado laboral; se deja de lado entonces a buena parte de los miembros de hogares con ingresos inferiores a la línea de pobreza.

Para el grupo de los empleados los indicadores de pobreza son menores que los de la población total, porque en los hogares pobres hay más dependencia económica, es decir, la relación empleados/ miembros del hogar es menor. Como muestra el cuadro 2 , en promedio trabaja uno de cada tres miembros de hogares pobres, mientras que en los no pobres trabaja uno de cada dos. Además, los primeros son más numerosos; mientras que el $65 \%$ de ellos tiene más de tres miembros, el $60 \%$ de los no pobres tiene tres o menos (cuadro 3). Por ello, cuando la población de referencia es la de los trabajadores (empleados) se toman menos individuos por hogar pobre y se incluye a la mitad de los miembros de hogares con ingreso superior al umbral de pobreza.

La gráfica 1 muestra que en los primeros años de la década analizada la leve reducción en los indicadores de pobreza se presentó en la misma dimensión para el total de la población y para los trabajadores. La variación porcentual de los índices de pobreza entre 2002 y 2004 es igual en los trabajadores y en el total de la población. A partir de 2004 (y con la misma metodología utilizada hasta 2005) empieza a presentarse una reducción más grande en los indicadores de la pobreza

\footnotetext{
${ }^{4}$ Los intervalos de confianza indican que la reducción año a año de todos los indicadores de pobreza es estadísticamente significativa.
}

Revista de Economía Institucional, vol. i7, N. ${ }^{\circ} 32$, Primer Semestre/2oi5, Pp. 295-3i5 
Cuadro 1

Indicadores de pobreza, población total y empleada, 2002-2012

\begin{tabular}{|c|c|c|c|c|c|c|c|c|}
\hline Indicador & \multicolumn{2}{|c|}{ Incidencia } & \multicolumn{2}{|c|}{ Brecha } & \multicolumn{2}{|c|}{ Intensidad } & \multicolumn{2}{|c|}{ Indicador de Sen } \\
\hline Población & Total & Empleada & Total & Empleada & Total & Empleada & Total & Empleada \\
\hline & 49,7 & 39,4 & 22,0 & 16,0 & 12,9 & & 29,9 & 22,6 \\
\hline 2002 & $\begin{array}{c}(49,3- \\
50,1)\end{array}$ & $\begin{array}{c}(38,7- \\
40,0)\end{array}$ & $\begin{array}{l}(21,8- \\
22,3)\end{array}$ & $\begin{array}{c}(15,8- \\
16,2)\end{array}$ & $\begin{array}{c}(12,7- \\
13,0)\end{array}$ & $\begin{array}{c}8,8 \\
(8,6-9,0)\end{array}$ & $\begin{array}{c}(29,7- \\
30,2)\end{array}$ & $\begin{array}{l}(22,3- \\
23,0)\end{array}$ \\
\hline & 48,4 & 38,5 & 20,8 & 15,2 & 11,8 & & 28,7 & 21,9 \\
\hline 2003 & $\begin{array}{c}(48,1- \\
48,7)\end{array}$ & $\begin{array}{c}(38,0- \\
39,0)\end{array}$ & $\begin{array}{l}(20,6- \\
21,0)\end{array}$ & $\begin{array}{c}(15,0- \\
15,5)\end{array}$ & $\begin{array}{c}(11,7- \\
12,0)\end{array}$ & $\begin{array}{c}8,2 \\
(8,0-8,4)\end{array}$ & $\begin{array}{c}(28,4- \\
28,9)\end{array}$ & $\begin{array}{l}(21,6- \\
22,1)\end{array}$ \\
\hline & 47,50 & 37,5 & 20,0 & 14,5 & 11,2 & & 27,9 & 21,1 \\
\hline 2004 & $\begin{array}{c}(47,2- \\
47,8)\end{array}$ & $\begin{array}{c}(37,1- \\
37,8)\end{array}$ & $\begin{array}{c}(19,9- \\
20,2)\end{array}$ & $\begin{array}{c}(14,3- \\
14,7)\end{array}$ & $\begin{array}{c}(11,1- \\
11,3)\end{array}$ & $\begin{array}{c}7,7 \\
(7,5-7,8)\end{array}$ & $\begin{array}{c}(27,7- \\
28,0)\end{array}$ & $\begin{array}{l}(20,8- \\
21,3)\end{array}$ \\
\hline 2005 & $\begin{array}{c}45,0 \\
(44,8- \\
45,3)\end{array}$ & $\begin{array}{c}34,6 \\
(34,2- \\
34,9)\end{array}$ & $\begin{array}{c}18,5 \\
(18,4- \\
18,6)\end{array}$ & $\begin{array}{c}13,0 \\
(12,8- \\
13,1)\end{array}$ & $\begin{array}{c}10,2 \\
(10,1- \\
10,3)\end{array}$ & $\begin{array}{c}6,7 \\
(6,6-6,9)\end{array}$ & $\begin{array}{c}26,1 \\
(25,9- \\
26,2)\end{array}$ & $\begin{array}{c}19,1 \\
(18,9- \\
19,4)\end{array}$ \\
\hline 2008 & $\begin{array}{c}42,2 \\
(41,9- \\
42,4)\end{array}$ & $\begin{array}{c}30,4 \\
(30,0- \\
30,7)\end{array}$ & $\begin{array}{c}18,4 \\
(18,3- \\
18,5)\end{array}$ & $\begin{array}{c}11,7 \\
(11,5- \\
11,9)\end{array}$ & $\begin{array}{c}10,8 \\
(10,7- \\
10,9)\end{array}$ & $\begin{array}{c}6,2 \\
(6,1-6,4)\end{array}$ & $\begin{array}{c}25,2 \\
(25,0- \\
25,3)\end{array}$ & $\begin{array}{c}17,0 \\
(16,8- \\
17,2)\end{array}$ \\
\hline 2009 & $\begin{array}{c}40,3 \\
(40,0- \\
40,6)\end{array}$ & $\begin{array}{c}29,5 \\
(29,1- \\
29,8)\end{array}$ & $\begin{array}{c}16,8 \\
(16,7- \\
16,9)\end{array}$ & $\begin{array}{c}11,0 \\
(10,9- \\
11,2)\end{array}$ & $\begin{array}{c}9,5 \\
(9,4-9,6)\end{array}$ & $\begin{array}{c}5,8 \\
(5,7-5,9)\end{array}$ & $\begin{array}{c}23,5 \\
(23,3- \\
23,7)\end{array}$ & $\begin{array}{c}16,3 \\
(16,1- \\
16,5)\end{array}$ \\
\hline 2010 & $\begin{array}{c}37,4 \\
(37,2- \\
37,7)\end{array}$ & $\begin{array}{c}27,3 \\
(26,9- \\
27,6)\end{array}$ & $\begin{array}{c}15,4 \\
(15,2- \\
15,5)\end{array}$ & $\begin{array}{c}10,1 \\
(10,0- \\
10,3)\end{array}$ & $\begin{array}{c}8,7 \\
(8,6-8,7)\end{array}$ & $\begin{array}{c}5,3 \\
(5,2-5,4)\end{array}$ & $\begin{array}{r}21,7 \\
(21,5- \\
21,8)\end{array}$ & $\begin{array}{c}15,0 \\
(14,8- \\
15,2)\end{array}$ \\
\hline 2011 & $\begin{array}{c}34,3 \\
(34,1- \\
34,5)\end{array}$ & $\begin{array}{c}24,5 \\
(24,2- \\
24,8)\end{array}$ & $\begin{array}{c}13,6 \\
(13,5- \\
13,7)\end{array}$ & $\begin{array}{c}8,8 \\
(8,7-9,0)\end{array}$ & $\begin{array}{c}7,4 \\
(7,3-7,5)\end{array}$ & $\begin{array}{c}4,5 \\
(4,4-4,6)\end{array}$ & $\begin{array}{c}19,5 \\
(19,4- \\
19,6)\end{array}$ & $\begin{array}{c}13,3 \\
(13,1- \\
13,5)\end{array}$ \\
\hline 2012 & $\begin{array}{c}32,9 \\
(32,7- \\
33,1)\end{array}$ & $\begin{array}{c}23,6 \\
(23,2- \\
23,9)\end{array}$ & $\begin{array}{c}12,9 \\
(12,8- \\
13,1)\end{array}$ & $\begin{array}{c}8,4 \\
(8,2-8,5)\end{array}$ & $\begin{array}{c}7,1 \\
(7,0-7,1)\end{array}$ & $\begin{array}{c}4,3 \\
(4,2-4,4)\end{array}$ & $\begin{array}{c}18,6 \\
(18,5- \\
18,8)\end{array}$ & $\begin{array}{c}12,7 \\
(12,5- \\
12,9)\end{array}$ \\
\hline
\end{tabular}

Notas: 1. Datos anuales para el total nacional de cada año; 2. En las estimaciones se usan ponderaciones muestrales; 3. Los intervalos de confianza de cada indicador al nivel de $99 \%$ van entre paréntesis y se calcularon usando el método de remuestreo bootstrap.

Fuente: Encuestas de hogares de cada año (Dane, 2013b), elaboración propia.

Cuadro 2

Situación de los miembros de hogares pobres y no pobres

\begin{tabular}{lrrrr}
\hline & \multicolumn{2}{c}{ Número } & \multicolumn{2}{c}{ Porcentaje } \\
\cline { 2 - 5 } & \multicolumn{1}{c}{ No pobre } & Pobre & No pobre & Pobre \\
\hline Empleado & 15.740 .403 & 4.849 .368 & 52,29 & 32,87 \\
Desempleado & 1.435 .607 & 930.449 & 4,77 & 6,31 \\
Inactivo & 7.838 .619 & 4.669 .793 & 26,04 & 31,65 \\
Menor de 12 años & 5.086 .426 & 4.305 .403 & 16,90 & 29,18 \\
Total & 30.101 .055 & 14.755 .013 & 100,00 & 100,00 \\
\hline
\end{tabular}

Notas: 1. Cifras estimadas para el total nacional en 2012; 2 . En las estimaciones se usan ponderaciones muestrales.

Fuente: Dane (2013b), elaboración propia.

para el caso de los empleados. Entre 2005 y 2008, la tendencia que se observó entre 2004 y 2005 se acentuó, pues aumentó visiblemente la diferencia entre la reducción de los indicadores de pobreza de la población empleada y total; mientras que la brecha e intensidad de la pobreza de la población total aumentó, en el caso de los trabajadores se redujo. Sin embargo, cabe señalar que no hay información disponible para 2006 y 2007 (cuando se puso en marcha la nueva encuesta de hogares), y por ello en la gráfica 1 se omite un efecto acumulado 
de 3 años. Además, en medio hay un cambio metodológico en la imputación de ingresos que puede haber afectado la ampliación de la diferencia mencionada en la variación de los indicadores de la población empleada y total.

Cuadro 3

Número de miembros del hogar (Porcentaje)

\begin{tabular}{lcc}
\hline Número de miembros & Hogar pobre & Hogar no pobre \\
\hline 1 & 5,40 & 14,27 \\
2 & 11,05 & 20,85 \\
3 & 18,83 & 24,27 \\
4 & 23,78 & 20,62 \\
5 & 17,99 & 10,95 \\
6 & 10,50 & 4,95 \\
7 & 5,74 & 2,21 \\
8 & 3,07 & 1,01 \\
9 & 1,64 & 0,45 \\
10 & 0,89 & 0,22 \\
Más de 10 & 1,11 & 0,20 \\
Total & 100,00 & 100,00 \\
\hline
\end{tabular}

Notas: 1. Cifras estimadas para el total nacional de 2012; 2. Estimaciones con ponderaciones muestrales.

Fuente: Dane (2013b), elaboración propia.

\section{Gráfica 1}

Índices de pobreza, población total y empleada

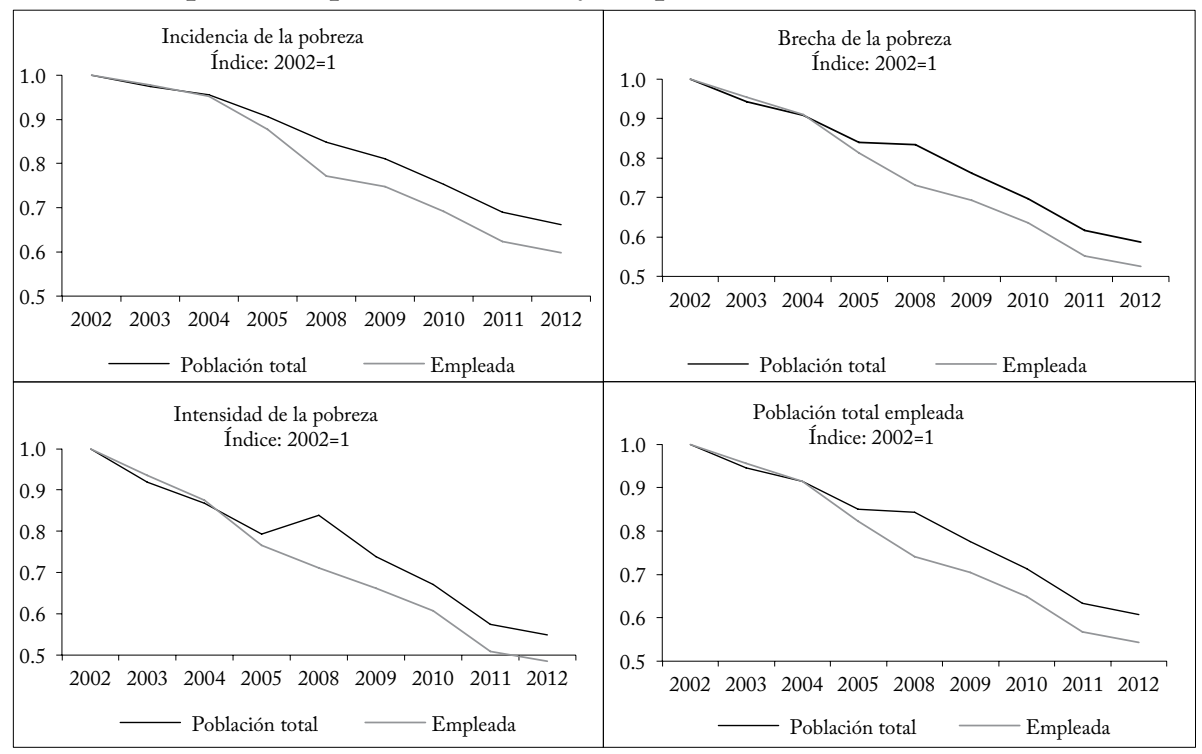

Índice $2002=1$.

Fuente: Dane (2013b), elaboración propia. 
La situación que se registró entre 2004 y 2008 (bien sea por el efecto de los cambios en la población, que impulsó la reducción de la pobreza, o por el cambio metodológico en 2006), contrasta con lo que sucede desde 2008; una reducción similar de los indicadores de pobreza de la población total y de los empleados (que en la gráfica 1 está representada por la similar pendiente de los índices). Desde 2008 hasta 2011 el indicador de intensidad se ha reducido más para el total de población que para los empleados, quizá debido a la ampliación del programa de transferencias condicionadas que mejoró el ingreso de los más pobres, pero no los sacó de la pobreza.

\section{DISTRIBUCIÓN GEOGRÁFICA DE LOS TRABAJADORES POBRES}

\section{INDICADORES DE POBREZA DE LOS TRABAJADORES POR ÁREA GEOGRÁFICA}

Todos los indicadores de pobreza que analizamos se redujeron en todas las zonas del país entre 2002 y 2012, como muestra el cuadro 4, que los presenta por zona geográfica: las 13 ciudades principales y sus áreas metropolitanas, las ciudades más pequeñas ${ }^{5}$ y las áreas rurales.

Se observan grandes diferencias en los niveles de pobreza de los trabajadores por zona geográfica. En el país, en promedio, uno de cada cuatro trabajadores pertenece a un hogar bajo el umbral de pobreza, cifra que aumenta en las zonas rurales, donde el 39\% de los trabajadores es pobre.

La pobreza de quienes trabajan en zonas rurales es mucho mayor que la de quienes trabajan en zonas urbanas, según todos los indicadores ${ }^{6}$. La diferencia entre las zonas rurales y las ciudades es mayor en brecha e intensidad, es decir, cuando se toma en cuenta el ingreso y su distribución entre los trabajadores pobres, y no solo el número de trabajadores con un ingreso per cápita familiar inferior al umbral de pobreza. Esa diferencia no ha disminuido en los últimos años ${ }^{7}, \mathrm{y}$ de hecho en términos relativos ha aumentado con la mayor reducción de la pobreza en las ciudades frente al mejoramiento de los ingresos de los trabajadores rurales.

\footnotetext{
5 O resto urbano: zonas densamente pobladas dentro del perímetro urbano municipal.

${ }^{6}$ La distribución de la población varía según el año, entre el 46\% y el $49 \%$ en las 13 ciudades principales, entre el $27 \%$ y el $29 \%$ en el resto urbano y entre el $22 \%$ y el $25 \%$ en zonas rurales.

${ }^{7}$ Como se muestra más adelante, la mayor diferencia entre la pobreza urbana y la rural en el caso de los empleados obedece más a las características del trabajo que a factores geográficos.
} 
Cuadro 4

Indicadores de pobreza de los trabajadores por zonas, 2002-2012

\begin{tabular}{|c|c|c|c|c|c|}
\hline Año & Indicador & $\begin{array}{c}\text { Principales } \\
\text { ciudades }\end{array}$ & Resto urbano & Rural & Total nacional \\
\hline \multirow{4}{*}{2002} & Incidencia & 25,75 & 46,18 & 56,07 & 39,37 \\
\hline & Brecha & 8,75 & 18,95 & 25,38 & 15,95 \\
\hline & $\begin{array}{l}\text { Intensidad } \\
\text { Indicador de }\end{array}$ & 4,28 & 10,39 & 14,92 & 8,77 \\
\hline & Sen & 12,06 & 25,27 & 33,09 & 22,60 \\
\hline \multirow{4}{*}{2003} & Incidencia & 26,30 & 44,53 & 53,18 & 38,49 \\
\hline & Brecha & 9,11 & 17,41 & 23,51 & 15,23 \\
\hline & $\begin{array}{l}\text { Intensidad } \\
\text { Indicador de }\end{array}$ & 4,49 & 9,15 & 13,61 & 8,20 \\
\hline & Sen & 12,47 & 23,27 & 31,05 & 21,61 \\
\hline \multirow{4}{*}{2004} & Incidencia & 24,39 & 43,91 & 53,83 & 37,45 \\
\hline & Brecha & 8,14 & 17,19 & 23,02 & 14,51 \\
\hline & $\begin{array}{l}\text { Intensidad } \\
\text { Indicador de }\end{array}$ & 3,93 & 8,99 & 12,95 & 7,68 \\
\hline & Sen & 11,22 & 22,91 & 30,48 & 20,06 \\
\hline \multirow{4}{*}{2005} & Incidencia & 22,42 & 42,56 & 48,06 & 34,56 \\
\hline & Brecha & 7,18 & 16,18 & 20,06 & 12,96 \\
\hline & $\begin{array}{l}\text { Intensidad } \\
\text { Indicador de }\end{array}$ & 3,34 & 8,36 & 11,13 & 6,72 \\
\hline & Sen & 9,92 & 21,75 & 26,69 & 19,13 \\
\hline \multirow{4}{*}{2008} & Incidencia & 17,93 & 37,97 & 48,01 & 30,37 \\
\hline & Brecha & 5,64 & 15,09 & 20,51 & 11,66 \\
\hline & $\begin{array}{l}\text { Intensidad } \\
\text { Indicador de }\end{array}$ & 2,66 & 8,10 & 11,73 & 6,24 \\
\hline & Sen & 7,88 & 20,21 & 27,39 & 17,01 \\
\hline \multirow{4}{*}{2009} & Incidencia & 16,73 & 38,14 & 45,06 & 29,47 \\
\hline & Brecha & 5,25 & 14,54 & 18,70 & 11,04 \\
\hline & $\begin{array}{l}\text { Intensidad } \\
\text { Indicador de }\end{array}$ & 2,49 & 7,60 & 10,42 & 5,80 \\
\hline & Sen & 7,35 & 19,61 & 25,67 & 16,31 \\
\hline \multirow{4}{*}{2010} & Incidencia & 15,12 & 35,02 & 42,82 & 27,26 \\
\hline & Brecha & 4,72 & 12,95 & 17,88 & 10,13 \\
\hline & $\begin{array}{l}\text { Intensidad } \\
\text { Indicador de }\end{array}$ & 2,25 & 6,62 & 10,09 & 5,32 \\
\hline & Sen & 6,64 & 17,53 & 23,98 & 15,02 \\
\hline \multirow{4}{*}{2011} & Incidencia & 13,18 & 32,45 & 38,36 & 24,53 \\
\hline & Brecha & 3,87 & 11,90 & 15,29 & 8,81 \\
\hline & $\begin{array}{l}\text { Intensidad } \\
\text { Indicador de }\end{array}$ & 1,75 & 5,99 & 8,23 & 4,46 \\
\hline & Sen & 5,45 & 16,05 & 20,48 & 13,30 \\
\hline \multirow{4}{*}{2012} & Incidencia & 12,01 & 30,65 & 38,86 & 23,55 \\
\hline & Brecha & 3,58 & 11,10 & 15,05 & 8,38 \\
\hline & $\begin{array}{l}\text { Intensidad } \\
\text { Indicador de }\end{array}$ & 1,64 & 5,59 & 8,03 & 4,25 \\
\hline & Sen & 5,05 & 15,06 & 20,36 & 12,72 \\
\hline
\end{tabular}

Fuente: encuestas de hogares de cada año (Dane, 2013b), elaboración propia.

\section{PARTICIPACIÓN DE LOS INDICADORES POR ZONA GEOGRÁFICA}

La gráfica 2 muestra la participación por zona geográfica en los indicadores de pobreza de los trabajadores para cada año. E1 37,5\% de los trabajadores pobres vive en zonas rurales, el 24,5\% en las 13 ciudades principales y el restante $38 \%$ en el resto urbano. Según el 
año y el indicador considerados, la participación de las ciudades oscila entre el $18,6 \%$ y el $31,2 \%$, la del resto urbano entre el $31,2 \%$ y el $39,4 \%{ }^{8}$, y la de las zonas rurales entre el $34,8 \%$ y el $45,0 \%$. En estas últimas se observan los mayores grados de brecha e intensidad. En 2002 , la participación en esos indicadores era del $42,1 \%$ y del $45 \%$, y en 2012 del 40,8\% y del 43\%; es decir que, a pesar de la reducción de la pobreza, las diferencias entre el sector rural y el urbano en lo que atañe a profundidad de la pobreza no variaron. Las zonas rurales continúan integrando gran parte de la pobreza de los trabajadores; en el periodo considerando no hubo variaciones importantes en la participación en sus indicadores de pobreza, como sí las hubo en las zonas urbanas (gráfica 2).

A pesar de que los indicadores de pobreza de los empleados son más bajos, en las ciudades principales hay una alta participación de los indicadores de pobreza pues estas concentran casi la mitad de los empleados del país, aunque la participación de cada indicador difiere, lo que indica diferencias en la problemática de la pobreza. La mayor participación se registra en la incidencia mientras que en la intensidad es baja; es decir, los ingresos de los trabajadores pobres son mayores respecto al umbral de pobreza en las ciudades que en las zonas rurales. La situación es diferente en el resto urbano donde todos los indicadores tienen una participación muy similar, intermedia entre los del campo y los de las ciudades principales.

\section{Gráfica 2}

Participación en los indicadores de pobreza de los trabajadores, por zona geográfica 2002-2012

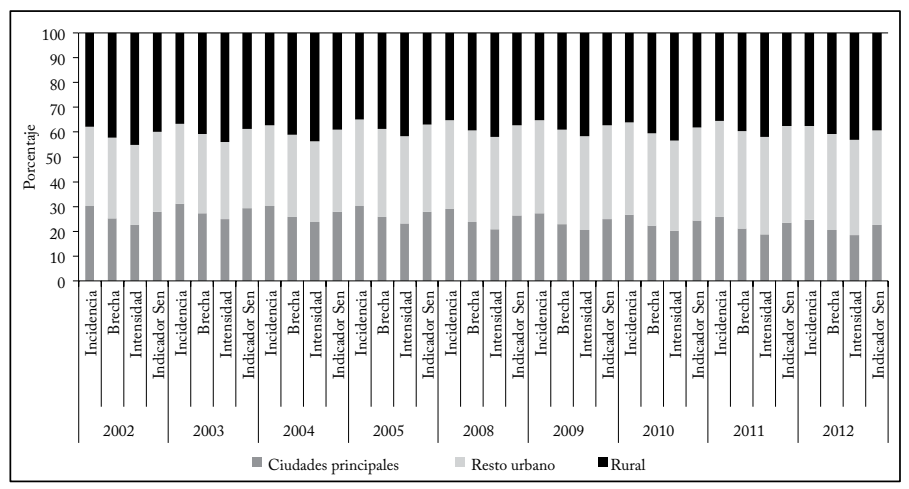

Fuente: Dane (2013b), elaboración propia.

\footnotetext{
${ }^{8}$ En esta zona, a diferencia de las otras, la participación en todos los indicadores de pobreza es similar, del 32\% en 2002 y del 38\% en 2012.
} 
La reducción de la pobreza de los trabajadores de las ciudades principales fue mayor que en el resto urbano y en el campo, y así lo indica la reducción estadísticamente significativa de la participación de las ciudades en todos los indicadores. Aunque esa reducción fue compensada por el incremento de la participación de las zonas urbanas de menor tamaño: como muestra la gráfica 2, es allí donde más aumentó la participación de todos los indicadores, del 32\% en 2002 al $38 \%$ en 2012.

\section{CARACTERIZACIÓN DE LOS TRABAJADORES POBRESY CAMBIOS EN LA ÚLTIMA DÉCADA}

Los trabajadores de hogares pobres tienen características personales que los diferencian de los trabajadores de hogares cuyo ingreso per cápita supera el umbral de pobreza, como muestra el cuadro 5 que compara sus características sociodemográficas, ocupacionales y de inserción laboral. En casi todos estos aspectos las diferencias son estadísticamente significativas.

Hay una mayor proporción de empleados hombres de hogares pobres. La diferencia en la participación de los hombres entre trabajadores pobres y no pobres era del $8 \%$ en 2002 , la cual se redujo en 2012 al 4\%. El aumento de la participación laboral femenina se reflejó en un mayor porcentaje de trabajadoras de hogares pobres; gran parte de las mujeres (jefas de hogar o cónyuges) que entraron al mercado laboral entre 2002 y 2012 tiene bajos ingresos (inferiores al umbral de pobreza dado el tamaño de su hogar). No hay grandes diferencias por grupos de edad (aunque son estadísticamente significativas) entre empleados de hogares pobres y no pobres: el único caso notable es el de los menores de 16 años de hogares pobres cuya participación es mayor; aunque esto quizá obedezca a la medición de la pobreza mediante el ingreso familiar per cápita, pues a medida que aumenta el tamaño del hogar aumenta la probabilidad de pobreza y, en general, ese aumento está asociado al número de menores en el hogar.

La principal diferencia en la composición de cada grupo de trabajadores por posición en el hogar se observa en los jefes de hogar y los cónyuges, donde están sobrerrepresentados los jefes de hogares pobres empleados, con una diferencia de 5 puntos porcentuales que se mantuvo en la última década; cabe destacar que la reducción de la participación laboral de miembros del hogar diferentes del jefe y su cónyuge es sido similar entre trabajadores pobres y no pobres. Como muestra el cuadro 5, el nivel educativo es la característica personal en 
Cuadro 5

Características personales y ocupacionales, trabajadores pobres y no pobres

\begin{tabular}{|c|c|c|c|c|c|c|}
\hline & \multicolumn{3}{|c|}{2002} & \multicolumn{3}{|c|}{2012} \\
\hline & Pobre & No pobre & Diferencia & Pobre & No pobre & Diferencia \\
\hline \multicolumn{7}{|l|}{ Sexo } \\
\hline Hombre & 66,18 & 58,05 & $8,13^{* * *}$ & 61,91 & 57,66 & $4,24^{* * * *}$ \\
\hline Mujer & 33,82 & 41,95 & $-8,13^{* * * * *}$ & 38,09 & 42,34 & $-4,24^{* * * *}$ \\
\hline \multicolumn{7}{|l|}{ Edad } \\
\hline Menor de 16 & 6,66 & 2,54 & $4,11^{* * *}$ & 6,68 & 2,10 & $4,57^{* * * *}$ \\
\hline $17-24$ & 17,55 & 17,07 & 0,48 & 16,31 & 15,78 & $0,53^{* * *}$ \\
\hline $25-35$ & 29,29 & 29,16 & 0,13 & 25,92 & 27,83 & $-1,91^{* * * *}$ \\
\hline $36-50$ & 31,28 & 33,77 & $-2,49^{* * *}$ & 32,13 & 32,63 & $-0,51^{*}$ \\
\hline \multirow{2}{*}{\multicolumn{7}{|c|}{ Nivel educativo }} \\
\hline & & & & & & \\
\hline Primaria & 60,70 & 29,78 & $30,92^{* * * *}$ & 55,38 & 27,55 & $27,83^{* * * *}$ \\
\hline Secundaria & 36,32 & 43,89 & $-7,57^{* * * * *}$ & 40,67 & 43,38 & $-2,71^{* * * *}$ \\
\hline Técnica y tecnológica & 2,02 & 22,07 & $-20,05^{* * * *}$ & 3,30 & 15,93 & $-12,62^{* * *}$ \\
\hline Profesional & 0,96 & 4,26 & $-3,30^{* * * *}$ & 0,64 & 13,14 & $-12,49 * * *$ \\
\hline \multicolumn{7}{|l|}{ Posición en el hogar } \\
\hline Jefe & 48,22 & 43,87 & $4,35^{* * * *}$ & 51,48 & 46,63 & $4,85^{* * * *}$ \\
\hline Cónyuge & 15,94 & 18,93 & $-2,99^{* * * *}$ & 16,95 & 20,10 & $-3,16^{* * * *}$ \\
\hline Hijo & 26,30 & 24,64 & $1,66^{* * * *}$ & 23,31 & 22,80 & 0,50 * \\
\hline Otro miembro & 9,53 & 12,55 & $-3,02^{* * * *}$ & 8,25 & 10,46 & $-2,20^{* * * * 1}$ \\
\hline \multicolumn{7}{|c|}{ Intensidad de la ocupación } \\
\hline Menos de 20 horas & 18,00 & 10,95 & $7,05^{* * * *}$ & 19,78 & 10,81 & $8,97^{* * * *}$ \\
\hline 20-35 horas & 15,64 & 12,40 & $3,24^{* * * *}$ & 17,99 & 10,66 & $7,33^{* * * *}$ \\
\hline $35-48$ horas & 30,84 & 40,38 & $-9,54 * * *$ & 33,68 & 44,02 & $-10,34^{* * * *}$ \\
\hline Más de 48 horas & 35,52 & 36,26 & $-0,74^{*}$ & 28,55 & 34,52 & $-5,96^{* * * * *}$ \\
\hline \multicolumn{7}{|l|}{ Segunda ocupación } \\
\hline Sí & 3,29 & 4,49 & $-1,20^{\text {**** }}$ & 9,95 & 8,54 & $1,41^{* * * *}$ \\
\hline No & 96,71 & 95,51 & $1,20^{* * * *}$ & 90,64 & 91,63 & $-1,41^{* * * *}$ \\
\hline \multicolumn{7}{|l|}{ Posición ocupacional } \\
\hline Asalariado & 24,96 & 49,04 & $-24,08^{* * * *}$ & 16,79 & 45,98 & $-29,19^{* * * *}$ \\
\hline Empleado doméstico & 11,33 & 9,37 & $1,96^{* * * *}$ & 8,20 & 6,90 & $1,30^{* * * *}$ \\
\hline Cuenta propia & 51,03 & 31,24 & $19,79^{\text {**** }}$ & 61,41 & 37,34 & $24,08^{* * * *}$ \\
\hline Patrón o empleador & 2,60 & 6,44 & $-3,85^{* * * *}$ & 2,36 & 5,74 & $-3,38^{* * * *}$ \\
\hline No remunerado & 10,09 & 3,91 & $6,18^{* * * * *}$ & 11,08 & 3,97 & $7,11^{* * * *}$ \\
\hline \multicolumn{7}{|l|}{ Subempleo horario } \\
\hline Sí & 20,74 & 15,46 & $5,28^{* * * * *}$ & 13,69 & 8,95 & $4,75^{* * * * *}$ \\
\hline \multirow{2}{*}{\multicolumn{7}{|c|}{ Subempleo calificación }} \\
\hline & & & & & & \\
\hline Sí & 2,25 & 3,82 & $-1,57^{* * * *}$ & 19,51 & 16,77 & $2,75^{* * * *}$ \\
\hline No & 97,75 & 96,18 & $1,57^{* * * *}$ & 80,49 & 83,23 & $-2,75^{* * * * *}$ \\
\hline \multicolumn{7}{|l|}{ Subempleo ingreso } \\
\hline Sí & 41,38 & 24,60 & $16,77^{* * * *}$ & 39,08 & 25,73 & $13,35^{* * * * *}$ \\
\hline No & 58,62 & 75,40 & $-16,77^{* * * *}$ & 60,92 & 74,27 & $-13,35^{* * * *}$ \\
\hline \multicolumn{7}{|l|}{ Rama de actividad } \\
\hline Agricultura & 33,70 & 14,41 & $19,30^{\text {***** }}$ & 32,93 & 13,92 & $19,01^{* * * 1 *}$ \\
\hline Minería & 1,14 & 0,78 & $0,35^{* * * * *}$ & 1,27 & 1,01 & $0,26^{* * * 1}$ \\
\hline Manufactura & 11,49 & 14,74 & $-3,24^{* * * * *}$ & 11,04 & 13,74 & $-2,71^{* * * 1}$ \\
\hline Construcción & 5,52 & 3,91 & $1,61^{* * * *}$ & 6,34 & 5,78 & $0,56^{* * * * 1}$ \\
\hline Comercio & 24,54 & 25,52 & $-0,98^{* * * *}$ & 25,26 & 26,97 & $-1,71^{\text {****** }}$ \\
\hline Servicios & 23,36 & 40,08 & $-16,73^{* * * *}$ & 23,16 & 38,57 & $-15,41^{* * * * *}$ \\
\hline
\end{tabular}

Nota: significancia estadística de las estimaciones de la diferencia en proporciones: ${ }^{* * *} \mathrm{p}<0,01 ;{ }^{* *} \mathrm{p}<0,05 ;{ }^{*} \mathrm{p}<0,10$. Fuente: encuestas de hogares de cada año (Dane, 2013b), elaboración propia.

la que más difieren los trabajadores pobres y no pobres; en 2012 más de la mitad de los pobres tiene como máximo educación primaria, solo el 3\% tiene educación técnica o tecnológica, y prácticamente 
no hay profesionales; estas últimas cifras poco variaron en la última década. El aumento del nivel educativo de los trabajadores se ha reflejado en forma diferenciada según el nivel de ingresos del hogar al que pertenecen; el leve aumento del nivel educativo promedio de los trabajadores pobres ocurrió en los niveles bajos, donde una fracción pasó de primaria a secundaria, mientras que el 9\% de los trabajadores no pobres hizo el tránsito de la educación técnica a la profesional.

En lo que respecta a las características de la ocupación de los trabajadores pobres y no pobres, no hay diferencias cuantitativas importantes (aunque sí estadísticas) en la antigüedad ni en la segunda ocupación ${ }^{9}$. Sin embargo, el aumento de la pluriactividad -más de una ocupación remunerada- es un cambio de la última década que ha afectado a trabajadores pobres y no pobres, con un mayor aumento en el primer grupo.

Hay notorias diferencias en la intensidad de la ocupación, la posición ocupacional y el subempleo. En promedio, los trabajadores pobres laboran menos horas por semana que los no pobres, y esta diferencia se amplió en los últimos años, cuando también se redujo la pobreza en buena proporción; esto indicaría (sin tener evidencia causal) que al tiempo que se redujo la pobreza aumentó la proporción de trabajadores pobres que laboran pocas horas por semana dentro de los empleados de hogares pobres; es decir, los que suelen salir de la pobreza trabajan más horas por semana y los que se mantienen en la pobreza tienen trabajos de baja intensidad horaria; el número de empleados pobres que trabajan menos de 20 horas aumentó en un 50\% entre 2008 y 2012, aunque la pobreza de los empleados se redujo. El subempleo es mayor entre los trabajadores pobres considerando la intensidad horaria ${ }^{10}$, y mucho más considerando el ingreso. No sucede así si se considera el subempleo por calificación, que aumentó notablemente, lo que está vinculado al bajo nivel educativo de los trabajadores pobres.

La posición ocupacional de los trabajadores pobres (frente a los de hogares no pobres) revela que las ocupaciones típicamente informales ${ }^{11}$ están asociadas a un bajo nivel de ingresos y a la pobreza ${ }^{12}$. En 2012 , el 61,5\% de los trabajadores pobres laboraba por cuenta propia,

\footnotetext{
${ }^{9}$ Los datos de antigüedad solo se tienen en forma continua desde 2008 y no se presentan en el cuadro 5. La diferencia mencionada se estimó para 2012.

${ }^{10} \mathrm{Un}$ subempleado por ingreso, calificación u horario es aquel que dice querer cambiar de ocupación por el motivo respectivo y está disponible para ocupar otro empleo. En el subempleo horario debe, además, trabajar menos de 48 horas a la semana.

${ }_{11}$ Sector/actividad/empleo, ver Portes y Haller (2004) y Perry et al. (2007).

${ }^{12}$ Ver Cecchini y Uthoff (2008) y Beccaria y Groisman (2008).
} 
casi un $20 \%$ no era remunerado o era empleado doméstico, y solo el $16,8 \%$ era asalariado. Los cambios en la composición ocupacional del mercado laboral -ampliación del trabajo por cuenta propia y reducción del trabajo asalariado- afectaron más a los trabajadores pobres, entre los cuales la participación de los asalariados se redujo en un $33 \%$. Los trabajadores por cuenta propia informales y los empleados domésticos tienen, en promedio, los ingresos laborales más bajos de todos los ocupados remunerados; en su mayoría terminan empleándose en estas actividades para sobrevivir y como una salida a los problemas de desempleo, e incluso a las malas condiciones de trabajo en empleos dependientes. Dadas las insuficientes oportunidades de empleo asalariado formal, deben crear sus propias oportunidades de empleo independiente, obteniendo ingresos insuficientes para suplir sus necesidades básicas (Fields, 2012).

El análisis del tamaño de la empresa confirma la asociación entre ser trabajador pobre y el tipo de ocupación; la mayoría de los trabajadores pobres desempeña actividades en las que no hay dependencia y cuando existe se presenta en pequeños establecimientos (sector informal); en 2012 solo el 5,5\% de ellos trabajaba en empresas con más de 30 empleados; entre los no pobres esa proporción era del $30 \%{ }^{13}$.

E1 sector de actividad indica la ubicación de los trabajadores pobres en la estructura productiva. Como ya se observó, la pobreza es mucho mayor en las zonas rurales, y esto se refleja en el hecho de que uno de cada tres trabajadores pobres trabaja en la agricultura. En las zonas urbanas, un elevado número de trabajadores pobres se dedica al comercio, actividad con gran participación de trabajadores por cuenta propia;, también es alta la proporción de trabajadores pobres en la construcción, donde las relaciones laborales suelen ser de carácter informal.

La probabilidad de ser trabajador pobre se estimó en función de variables sociodemográficas, ocupacionales, rama de actividad y zona geográfica, para determinar el tipo de vínculo y el peso de estos rasgos en la probabilidad de que un trabajador pertenezca a un hogar pobre. Se hicieron dos estimaciones para 2002 y 2012, una con un modelo lineal y otra con un modelo probit para verificar la consistencia de los resultados. Los resultados se presentan en el cuadro 6. La dirección de los efectos marginales de todas las variables coincide en ambos modelos, y en la mayoría de las variables el efecto marginal del modelo

\footnotetext{
13 Igual que en el caso de la antigüedad, no se tienen datos del tamaño del establecimiento para todos los meses de 2002, por ello esa información no se incluye en el cuadro 5.
} 
lineal (coeficiente estimado) es similar al del modelo probit (calculado en las medias de las variables explicativas).

La dirección del efecto de la edad y el sexo cambia en las estimaciones de 2002 y 2012, pero el efecto de la edad solo es significativo en las estimaciones de 2002, y es muy bajo. El efecto del sexo también cambia, y además de ser muy bajo solo es significativo al $5 \%$ en la estimación lineal de 2012. Estos resultados indican que son otras características personales las que más influyen en que los hogares de los trabajadores sean pobres; entre ellas el bajo nivel educativo, ser jefe de hogar, trabajar por cuenta propia o sin remuneración, trabajar pocas horas a la semana, trabajar en la agricultura o la construcción, y vivir en pueblos pequeños o en áreas rurales. El cuadro 6 muestra que estos factores tienen efectos marginales apreciables y significativos en ambos modelos.

Entre 2002 y 2012 se redujo el efecto del nivel de educación en la probabilidad de que un empleado sea pobre; en 2012 un bajo nivel de educación tenía mayor correlación con la pertenencia a un hogar pobre, y aunque en 2012 es menor (por la mayor escolaridad de toda la población), sigue siendo un aspecto distintivo de los trabajadores pobres; en promedio, un año adicional de educación, ceteris paribus, reduce la probabilidad de ser trabajador pobre entre 0,0261 y 0,0323. Como ya se indicó (cuadros 2 y 5), en los hogares pobres hay mayor dependencia económica y el 51,5\% de los trabajadores de estos hogares son jefes de hogar; de allí la alta asociación entre ser jefe de hogar y hogar pobre. Esa asociación aumentó en comparación con la de ser cónyuge y se redujo frente a la de otros miembros del hogar.

Aunque el efecto de trabajar por cuenta propia o sin remuneración se redujo en esos diez años, son las características ocupacionales más correlacionadas con la pobreza de los trabajadores, como indicaba el hecho de que más del $70 \%$ de los trabajadores pobres sean por cuenta propia o no remunerados. En el periodo de estudio no varió el efecto, ceteris paribus, del sector de actividad; la agricultura y la construcción siguen siendo los sectores donde es mayor la probabilidad de ser trabajador de un hogar pobre tomando como referencia la industria manufacturera.

También se mostró que el número de trabajadores pobres que labora menos de 20 horas semanales aumentó un 50\% entre 2008 y 2012, y que gran parte de los que superaron el umbral de pobreza trabajaban tiempo completo o con alta intensidad horaria. Ese hecho se refleja en el cambio de magnitud del efecto marginal del número de horas (manteniendo el signo negativo, es decir, un mayor número 
de horas reduce la probabilidad de ser trabajador pobre); en el modelo lineal, ese efecto se multiplicó por cuatro y en el modelo probit por tres, lo que indica que cuanto más baja es la intensidad horaria mayor es la probabilidad de ser miembro de un hogar pobre, efecto que ha adquirido mayor relevancia.

Cuadro 6

Efectos marginales sobre la probabilidad de ser trabajador pobre

\begin{tabular}{|c|c|c|c|c|}
\hline & \multicolumn{2}{|c|}{2002} & \multicolumn{2}{|c|}{2012} \\
\hline & Modelo lineal & Modelo probit & Modelo lineal & Modelo probit \\
\hline \multicolumn{5}{|l|}{ Características sociodemográficas } \\
\hline Sexo $(1=$ hombre $)$ & 0,00206 & 0,00242 & $-0,00700^{* *}$ & $-0,00545$ \\
\hline Edad & $-0,00258^{* * * *}$ & $-0,00374^{* * * *}$ & 0,00076 & $-0,00013$ \\
\hline Edad2 & $-0,00003^{* * *}$ & $-0,00004^{* * *}$ & $-0,00007^{* * *}$ & $-0,00007^{* * *}$ \\
\hline Escolaridad (años) & $-0,03238^{* * *}$ & $-0,04355^{* * * *}$ & $-0,02609^{\text {***** }}$ & $-0,03228^{* * *}$ \\
\hline Cónyuge & $-0,05964^{* * *}$ & $-0,07736^{\text {**** }}$ & $-0,07994^{* * * * *}$ & $-0,09306^{* * *}$ \\
\hline Hijo & $-0,06599^{* * * *}$ & $-0,07776^{\text {***** }}$ & $-0,06336^{\text {***** }}$ & $-0,07095^{* * *}$ \\
\hline Otros & $-0,11201^{* * * *}$ & $-0,13028^{\text {***** }}$ & $-0,09021^{* * * *}$ & $-0,09757^{* * *}$ \\
\hline \multicolumn{5}{|l|}{ Caracteristicas ocupacionales } \\
\hline Horas de trabajo (a la semana) & $-0,00035^{* * *}$ & $-0,00039^{\text {***** }}$ & $-0,00136^{* * * *}$ & $-0,00122^{* * *}$ \\
\hline Empleados domésticos & $-0,01890^{* *}$ & $-0,01129$ & $-0,00572$ & $-0,00110$ \\
\hline Por cuenta propia & $0,14930^{* * *}$ & $0,17584^{* * * *}$ & $0,10435^{\text {***** }}$ & $0,11107^{* * *}$ \\
\hline Empleador & $-0,03410^{* * * *}$ & $-0,05996^{* * * * *}$ & $-0,06596^{* * * *}$ & $-0,09847^{* * * *}$ \\
\hline No remunerado & $0,20292^{* * *}$ & $0,22258^{\text {**** }}$ & $0,15819^{\text {***** }}$ & $0,15361^{* * * *}$ \\
\hline Subempleo horario & $0,02991^{* * *}$ & $0,03617^{\text {**** }}$ & $0,03826^{\text {***** }}$ & $0,04236^{* * * *}$ \\
\hline Subempleo por calificación & $-0,04720^{* * * *}$ & $-0,03411^{\text {***** }}$ & $-0,03938^{* * * *}$ & $-0,02307^{\text {**** }}$ \\
\hline Subempleo por ingreso & $0,10930^{* * *}$ & $0,12413^{* * * *}$ & $0,10403^{* * * *}$ & $0,09653^{* * * *}$ \\
\hline \multicolumn{5}{|l|}{ Rama de actividad } \\
\hline Agrícola & $0,07578^{* * *}$ & $0,05859^{\text {**** }}$ & $0,08649^{\text {**** }}$ & $0,05721^{* * *}$ \\
\hline Construcción & $0,05022^{* * *}$ & $0,04332^{* * * *}$ & $0,03416^{\text {***** }}$ & $0,02753^{* * *}$ \\
\hline Comercio & $-0,01732^{* * * *}$ & $-0,02072 * * * *$ & $-0,01878 * * *$ & $-0,02270^{* * * *}$ \\
\hline Servicios & $-0,01394^{* *}$ & $-0,03597^{* * *}$ & $-0,00197$ & $-0,01485^{* * * *}$ \\
\hline \multicolumn{5}{|l|}{ Región } \\
\hline Resto urbano & $0,11275^{* * *}$ & $0,13678^{* * * *}$ & $0,13901^{* * * *}$ & $0,15500^{* * *}$ \\
\hline Rural & $0,04711^{* * * *}$ & $0,04385^{\text {***** }}$ & $0,02413^{* * * *}$ & $0,02634^{* * *}$ \\
\hline
\end{tabular}

Notas: 1. En las dummy de posición en el hogar y ocupacional, rama de actividad y región, se tomó como referencia ser jefe de hogar, ser asalariado, la manufactura y las ciudades principales, respectivamente; 2 . Nivel de significatividad: ${ }^{* * *} \mathrm{p}<0,01 ;{ }^{* *} \mathrm{p}<0,05 ;{ }^{*} \mathrm{p}<0,1 ; 3$. Los efectos marginales del modelo probit se calcularon en las medias de las variables explicativas.

Fuente: encuestas de hogares de cada año (Dane, 2013b), elaboración propia.

Un resultado interesante de las estimaciones es que la influencia de vivir en zonas rurales en la probabilidad de ser trabajador informal no es tan grande como se esperaría (dados los altos indicadores de pobreza de las zonas rurales frente a los de las zonas urbanas). Aún más notable es que el efecto marginal sobre la probabilidad de ser trabajador pobre (positivo tomando como referencia las ciudades principales), ceteris paribus, de vivir en el resto urbano sea de 4 a 5 veces mayor que el de vivir en el campo. Entre 2002 y 2012 el efecto de vivir en zonas rurales sobre la probabilidad de estar empleado y pertenecer a un hogar pobre se redujo, mientras que el de vivir en zonas urbanas pequeñas aumentó (frente al de vivir en grandes ciudades). Esto indicaría que la pobreza de los trabajadores rurales no 
se debe intrínsecamente a la zona donde viven sino a características personales (como el bajo nivel educativo) y de la ocupación (trabajar por cuenta propia o sin remuneración); y que la pobreza rural está más vinculada al tipo de actividad que desempeñan los trabajadores rurales que a la ubicación geográfica (el 65\% de ellos se dedica a labores agrícolas, el $64 \%$ trabaja por cuenta propia y el $17 \%$ en trabajos familiares no remunerados).

\section{CONCLUSIONES}

La pobreza se redujo sistemáticamente en el país entre 2002 y 2012, y aún más en el caso de los empleados, de modo que los hogares con menor dependencia económica fueron los que superaron el umbral de pobreza. Un factor de la pobreza de países en desarrollo es la falta de una protección social mínima que lleva a que los miembros de hogares pobres se empleen en trabajos de bajos salarios y baja productividad, informales o por cuenta propia. A diferencia de la relación entre desempleo y pobreza que se encuentra en los países de altos ingresos, en los países de América Latina la pobreza guarda mayor relación con el tipo de inserción laboral que con el desempleo (con matices entre países), aunque la tasa de desempleo es mucho mayor para los miembros de hogares pobres: en Colombia es del 16\% y la de hogares no pobres es del 9\%.

En Colombia, uno de cada cuatro trabajadores integra un hogar pobre, cifra que es mayor en las zonas rurales donde el 39\% de los trabajadores es pobre y el ingreso de los pobres con respecto al umbral de pobreza es más bajo que en las ciudades. Del total de trabajadores pobres, el 24,5\% vive en las 13 ciudades principales, el 38\% en el resto urbano y el $37,5 \%$ en zonas rurales. Los trabajadores de las zonas rurales tienen mayores niveles de pobreza sea cual sea el indicador; esta diferencia, que se ha mantenido en la última década, se debe principalmente al tipo de empleo (trabajo por cuenta propia, familiar o no remunerado) y al sector económico donde trabajan (agricultura).

Junto con la reducción de la pobreza de los trabajadores se redujo la influencia del nivel educativo y de algunos tipos de empleo (cuenta propia y no remunerado) en la probabilidad de ser trabajador pobre, y aumentó el efecto de las ocupaciones de baja intensidad horaria y del subempleo horario. No obstante, los trabajadores de hogares pobres mantienen ciertas características: bajo nivel educativo, vivir en pueblos pequeños y en zonas rurales, trabajar por cuenta propia o sin remuneración, trabajar pocas horas a la semana, y en el sector agrícola o en la construcción. Las actividades típicamente informales 
están asociadas a los niveles de pobreza dentro de los trabajadores; el $61,5 \%$ de los trabajadores pobres tiene empleos por cuenta propia, el $11 \%$ no es remunerado, el $8,2 \%$ es empleado doméstico y la mayor parte de los asalariados trabaja en establecimientos pequeños. Sin embargo, se necesita explorar con mayor profundidad el vínculo entre la pobreza y la inserción laboral de los trabajadores de hogares pobres en actividades y empleos informales.

\section{REFERENCIAS BIBLIOGRÁFICAS}

1. Alkire, S. y J. Foster. "Counting and multidimensional poverty measurement”, OPHI working paper series 32, Oxford, University of Oxford, 2009.

2. Beccaria, L. y F. Groisman. "Informalidad y pobreza en Argentina", Investigación Económica LXVII, 266, 2008, pp.135-169.

3. Banerjee, A. y E. Duflo. Poor economics, Nueva York, Public Affairs, 2011.

4. Cecchini, S. y A. Uthoff. "Poverty and employment in Latin America: 1990-2005”, Cepal Review 94, 2008, pp. 41-56.

5. Cepal. Panorama social de América Latina 2012, Cepal, Santiago de Chile, 2013.

6. Cruces, G. y L. Gasparini. "Políticas sociales para la reducción de la desigualdad y la pobreza en América Latina y el Caribe. Diagnóstico, propuesta y proyecciones en base a la experiencia reciente", documento de trabajo 142, CEDLAS, La Plata, 2013.

7. Dane. "Pobreza monetaria y multidimensional en Colombia 2012", Boletín de Prensa, Bogotá, 2013a.

8. Dane. "Gran Encuesta Integrada de Hogares. Microdatos anonimizados", 2013b, [www.dane.gov.co].

9. Fields, G. "Challenges and policy lessons for the growth-employmentpoverty nexus in developing countries", IZA Journal of Labor Policy 1, 6, 2012.

10. Foster, J.; J. Greer y E. Thorbecke. "A class of decomposable poverty measures”, Econometrica 52, 3, 1984, pp. 761-766.

11. Gasparini, L.; M. Cicowiez y W. Sosa E. Pobreza y desigualdad en América Latina. Conceptos, herramientas y aplicaciones, Buenos Aires, Temas, 2013.

12. GEEP. "Compendio de mejores prácticas en la medición de la pobreza", Grupo de Expertos en Estadísticas de Pobreza, Grupo de Río, Santiago de Chile, Cepal, 2007.

13. Majid, N. “The working poor in developing countries", International Labour Review 140, 3, 2001, pp.271-291.

14. Marx, I. y G. Verbist. "Low-paid work and poverty: A cross-country perspective”, S. Baze, M. Gregory y W. Salverda, eds., Low-wage employment in Europe, Cheltenham, Edward Elgar, 1998, pp. 63-86.

15. Medina, F. y M. Galván. "Crecimiento económico, pobreza y distribución del ingreso. Fundamentos teóricos y evidencia empírica para América Latina, 1997-2007”, Serie Estudios Estadísticos 82, Santiago de Chile, Cepal, 2014.

16. MESEP. "Pobreza monetaria en Colombia: nueva metodología y cifras 2002-2010”, Bogotá, Dane-DNP, 2012. 
17. Perry, G.; W. Maloney, O. Arias et al., Informalidad: escape y exclusión, Bogotá, Banco Mundial, 2007.

18. Ponthieux, S. y P. Concialdi. "Bajos salarios y trabajadores pobres: una comparación entre Francia y Estados Unidos”, Cuadernos de Relaciones Laborales 18, 2001, pp. 173-203.

19. Portes, A. y W. Haller. "La economía informal”, Santiago de Chile, Cepal, Serie políticas sociales No. 100, 2004.

20. Sánchez, R. "Balance y características del mercado de trabajo y generación de empleo en Colombia 2002-2010", Apuntes del Cenes 32, 55, 2013, pp. 93-124. 